\title{
Pretreatment neutrophil-to-lymphocyte ratio as a survival predictor for small-cell lung cancer
}

This article was published in the following Dove Press journal:

OncoTargets and Therapy

20 September 2016

Number of times this article has been viewed

\section{Xin Wang ${ }^{1,2, *}$ \\ Feifei Teng ${ }^{2,3, *}$ \\ Li Kong ${ }^{2}$ \\ Jinming $\mathrm{Yu}^{2}$}

'School of Medicine and Life Sciences, University of Jinan-Shandong Academy of Medical Sciences, ${ }^{2}$ Department of Radiation Oncology, Shandong Cancer Hospital and Institute, ${ }^{3}$ School of Medicine, Shandong University, Jinan, Shandong Province, People's Republic of China

*These authors contributed equally to this work
Correspondence: Jinming Yu Department of Radiation Oncology, Shandong Cancer Hospital and Institute, Shandong University, No 440 Jiyan Road, Jinan, Shandong Province 250II7, People's Republic of China

Tel +8653 I 87984729

Fax +8653I 87984079

Email sdyujinming@163.com
Background: The inflammatory response indexes, neutrophil-to-lymphocyte ratio (NLR) and platelet-to-lymphocyte ratio (PLR), have prognostic value for a variety of cancers. However, their prognostic value for small-cell lung cancer (SCLC) has been rarely reported. In this study, we monitored changes of NLR and PLR along with the clinical outcomes in patients with limited-stage and extensive-stage SCLC who received standard treatments.

Materials and methods: We retrospectively reviewed the records of 153 patients who were pathologically diagnosed with SCLC and collected their hematological data at different time points during disease and treatment process. Kaplan-Meier analysis and Cox proportional hazards models were used to determine the prognostic significance of NLR and PLR for overall survival (OS) and progression-free survival (PFS).

Results: The median OS and PFS for all patients were 23.3 months and 11.0 months, respectively. After applying cutoffs of 3.2 for NLR and 122.7 for PLR, NLR, but not PLR, showed independent prognostic significance. High-NLR group was associated with shorter median OS (high vs low, 18.0 months vs 31.0 months, $P<0.01$ ) and shorter PFS (high vs low, 9.3 months vs 13.0 months, $P=0.006$ ). The cumulative 3 -year OS rate and 3 -year PFS rate of high-NLR group versus low-NLR group were $14.3 \%$ versus $37.3 \%$ and $8.6 \%$ versus $22.9 \%$, respectively. In the multivariate analysis, both disease stage and NLR at diagnosis were independent prognostic factors for OS and PFS.

Conclusion: The NLR at diagnosis showed significant prognostic value for clinical outcomes in SCLC patients treated with chemoradiotherapy. As an effective biomarker of host immune status, NLR could potentially help monitoring disease progression and adjusting treatment plans.

Keywords: small-cell lung cancer, neutrophil-to-lymphocyte ratio, platelet-to-lymphocyte ratio, chemoradiotherapy, thoracic radiation

\section{Introduction}

Small-cell lung cancer (SCLC) accounts for $\sim 15 \%-20 \%$ of all lung cancers. ${ }^{1}$ It is characterized by extremely aggressive biological nature, rapid growth, and early metastasis. Although SCLC is highly responsive to chemoradiotherapy (CRT) initially, it tends to recur and have devastating prognosis. The median survival time is only 15-20 months for limited-stage (LS) and 8-13 months for extensive-stage (ES) SCLC. ${ }^{2-4}$ Because of the aggressive biological nature and high mortality of SCLC, it might be helpful to have an effective biomarker that helps monitoring disease progression, adjusting treatment plans, and preventing overtreatment.

In the 19th century, Rudolf Virchow first observed leukocytes within tumors, indicating a possible link between tumor progression and inflammation. Afterward, tumor-associated inflammation was shown to play a critical role in tumor development, including tumor initiation, progression, transformation, invasion, and metastasis. ${ }^{5}$ 
Moreover, tumor-associated inflammation could inhibit host immune response and enhance genomic instability, which is an important cause for cancer initiation. de Visser et $\mathrm{al}^{6}$ illustrated that chronic inflammation disturbed the interactions between host immune cells because of abnormal cellular profiles, soluble mediators, and signal pathways. Further, the destructive circumstances lead to genomic instability and increased risk of cancer development. The most direct evidence of the association between cancer development and chronic system inflammation comes from patients treated with chronic inflammation inhibitors, who were prone to cancer progression before treatment and could achieve chemopreventative potential afterward. It has been reported that the inflammation inhibitors such as aspirin and selective cyclooxygenase-2 inhibitors could significantly decrease cancer risk. ${ }^{7}$ As a paraneoplastic surrogate index for host immune response and inflammation status, the prognostic value of neutrophil-to-lymphocyte ratio (NLR) and plateletto-lymphocyte ratio (PLR) has been demonstrated in a variety of cancers, including colorectal cancer, gastric cancer, esophageal cancer, non-small-cell lung cancer, breast cancer, ovarian cancer, and endometrial cancer, ${ }^{8-14}$ but it was rarely reported in SCLC. Therefore, in this study, we verified the prognostic value of NLR and PLR in SCLC patients and provide informative knowledge to the disease prognosis.

\section{Materials and methods} Patient characteristics

We retrospectively analyzed 153 patients who were diagnosed with SCLC between January 2009 and September 2013 in Shandong Cancer Hospital and Institute. The study protocols were approved by the Ethics Committee of Shandong Cancer Hospital and Institute, People's Republic of China. All participants provided written informed consent. All the included patients were pathologically diagnosed with SCLC by biopsy. The patients with LS SCLC received combined concurrent CRT or sequential CRT, and they received combination chemotherapy as first-line treatment for at least two cycles, which was based on platinum agents such as cisplatin and carboplatin. The radiation modes for all LS SCLC patients were conventional fraction radiotherapy and accelerated hyperfractionation, with the total dose of radical thoracic radiation (TRT) ranging from 45 Gy to 62 Gy. After radical TRT, patients who achieved complete response or nearly complete response received prophylactic cranial irradiation. The majority of the patients with ES SCLC received combined sequential CRT, and only a few of them received concurrent CRT. The radiation modes also included conventional fraction radiotherapy and accelerated hyperfractionation, with the total dose of palliative TRT ranging from 30 Gy to 60 Gy. Prophylactic cranial irradiation was implemented for patients who achieved high response rate. Computed tomography scan was used to evaluate treatment response based on evaluation criteria in solid tumors (RECIST) Version 1.1.15

\section{Data collection}

The clinical baseline data of patients' characteristics were obtained from the electronic medical record system of Shandong Cancer Hospital and Institute. The following parameters were recorded: demographics, smoking habits, disease stage, Karnofsky performance status, inflammation situation, granulocyte-macrophage colony-stimulating factor (GM-CSF), and radiation modes. Besides this, hemoglobin, albumin, lactate dehydrogenase, and alkaline phosphatase were evaluated at diagnosis. Absolute blood cell counts of neutrophils, lymphocytes, and platelets were collected at four time points, which were at diagnosis, after the first cycle of chemotherapy, after radiotherapy, and at disease progression. GM-CSF is often administered when absolute white blood cell count is $<3,000$ cells $/ \mathrm{mL}$. The NLR was defined as the absolute neutrophil count divided by the absolute lymphocyte count. The PLR was defined as the absolute platelet count divided by the absolute lymphocyte count.

\section{Statistical analysis}

The optimal cutoff values of NLR and PLR were set at where the receiver operating characteristic curve yielded the combined maximum of sensitivity plus specificity. Primary and secondary end points were overall survival (OS) and progression-free survival (PFS), respectively. OS was defined as the time from diagnosis to death, from any cause, or to the last follow-up date. PFS was defined as the time from diagnosis until evidence of disease progression, including thorax failure or distant metastasis. OS and PFS were performed using Kaplan-Meier method, and comparisons were carried out by log-rank test. Continuous variables of patients were summarized by mean values with standard deviation. The comparisons between high- and low-NLR/PLR groups were performed with MannWhitney $U$-test or Wilcoxon signed-rank test. Categorical variables were summarized by frequencies and analyzed with chi-square tests or two-sided Fisher's exact test. To determine the independent prognostic factors, a multivariate analysis was performed using Cox proportional hazards model, and hazard ratios were reported as relative risks with corresponding 95\% confidence intervals. All tests were two sided, and $P<0.05$ was considered statistically significant. 


\section{Results}

\section{Patient characteristics}

A total of 153 SCLC patients were treated with CRT during the study period. In the end, we had NLR and PLR data for all the patients at diagnosis, 145 patients after first cycle of chemotherapy and after radiotherapy and 69 patients at disease progression point. The basal levels of patient characteristics are shown in Table 1. Patients' median age at diagnosis was 62 years (range $28-79$ years). The median PFS was 11.0 months (1.2-67.7 months), and median OS was 23.3 months (6.0-70.2 months). Applying receiver operating characteristic analysis, the optimal cutoff values were 3.2 for NLR, with area under the curve (AUC) of 0.723 , and 122.7 for PLR, with AUC of 0.623 .

We also summarized the changes of NLR and PLR along with the progression of disease and treatment, as shown in Table 2. NLR decreased after one cycle of chemotherapy compared to the time of diagnosis ( $2.87 \pm 1.60$ vs $3.26 \pm 1.36$, $P=0.016$ ), and then it significantly increased after TRT $(8.98 \pm 9.96$ vs $3.26 \pm 1.36, P<0.01)$. At disease progression point, NLR decreased again but was still higher than the level at the time of diagnosis $(4.27 \pm 3.70$ vs $3.26 \pm 1.36, P=0.499)$.

In contrast, PLR increased after one cycle of chemotherapy compared to the time of diagnosis $(182.18 \pm 94.30 \mathrm{vs}$ $151.50 \pm 60.01, P<0.01$ ), and then it significantly increased again after TRT $(308.49 \pm 252.23$ vs $151.50 \pm 60.01, P<0.01)$. At disease progression point, PLR decreased but was still higher than the level at the time of diagnosis $(193.58 \pm 106.35$ vs $151.50 \pm 60.01, P=0.026$ ).

\section{Factors associated with NLR and PLR}

The associations between clinical features of patients and NLR or PLR values are summarized in Table 3 with NLRor PLR-based stratifications. From the comparisons, we found more ES SCLC patients in the high-NLR group $(P<0.01)$, which also had worse Karnofsky performance status $(P=0.043)$, lower serum albumin level $(P=0.005)$, lower response rate for initial chemotherapy $(P=0.027)$, lower prophylactic cranial irradiation rate $(P=0.004)$, less radiation therapy dose $(P=0.047)$, and lower second-line chemotherapy rate $(P=0.006)$. For PLR-based stratification, we also found more ES SCLC patients in the high-PLR group ( $P=0.009)$, which had lower response rate for initial chemotherapy $(P=0.041)$ and lower second-line chemotherapy rate $(P=0.038)$.

From Table 3, we could see that both NLR and PLR had predictive abilities for initial chemotherapy response. The response rate is $94.0 \%$ for low-NLR group versus $77.1 \%$
Table I Patient baseline characteristics

\begin{tabular}{|c|c|}
\hline Variables & $\begin{array}{l}\text { Number of } \\
\text { patients (\%) }\end{array}$ \\
\hline Age (range), years $(n=153)$ & $62(28-79)$ \\
\hline \multicolumn{2}{|l|}{$\operatorname{Sex}(n=153)$} \\
\hline Male & I I 8 (77.I) \\
\hline Female & 35 (22.9) \\
\hline \multicolumn{2}{|l|}{ Smoking status $(n=153)$} \\
\hline Never smoker & $47(30.7)$ \\
\hline Current or ex-smoker & $106(69.3)$ \\
\hline \multicolumn{2}{|l|}{ Stage $(n=153)$} \\
\hline LS & $86(56.2)$ \\
\hline ES & $67(43.8)$ \\
\hline \multicolumn{2}{|l|}{ KPS at diagnosis $(n=153)$} \\
\hline$\geq 80$ & $139(90.8)$ \\
\hline$<80$ & $14(19.2)$ \\
\hline \multicolumn{2}{|l|}{$\mathrm{Hb}$ at diagnosis $(\mathrm{n}=153)$} \\
\hline Normal ( $\leq$ ULN) & $146(95.4)$ \\
\hline Low $(<$ LLN $)$ & $7(4.6)$ \\
\hline \multicolumn{2}{|l|}{ Alb at diagnosis $(n=\mid 36)$} \\
\hline Normal $(\leq \mathrm{ULN})$ & I0I (74.3) \\
\hline Low $(<$ LLN) & $35(25.7)$ \\
\hline \multicolumn{2}{|l|}{$\mathrm{LDH}$ at diagnosis $(\mathrm{n}=136)$} \\
\hline Normal $(<\mathrm{ULN})$ & $74(54.4)$ \\
\hline High $(\geq \mathrm{ULN})$ & $62(45.6)$ \\
\hline \multicolumn{2}{|l|}{ ALP at diagnosis $(n=136)$} \\
\hline Normal $(<$ ULN) & $126(92.6)$ \\
\hline High $(\geq U L N)$ & $10(7.4)$ \\
\hline NLR at diagnosis, mean $\pm s d$ & $3.26 \pm 1.36$ \\
\hline PLR at diagnosis, mean $\pm s d$ & $151.50 \pm 60.01$ \\
\hline \multicolumn{2}{|l|}{ Response for initial chemotherapy $(n=153)$} \\
\hline $\mathrm{CR}$ & $9(5.9)$ \\
\hline PR & $|3|(85.6)$ \\
\hline SD & $5(3.3)$ \\
\hline PD & $8(5.2)$ \\
\hline \multicolumn{2}{|l|}{ Prophylactic cranial irradiation $(n=127)$} \\
\hline Yes & $30(23.6)$ \\
\hline No & $97(76.4)$ \\
\hline \multicolumn{2}{|l|}{ Inflammation at diagnosis $(n=153)$} \\
\hline Yes & $38(24.8)$ \\
\hline No & $115(75.2)$ \\
\hline \multicolumn{2}{|l|}{ Stimulate neutrophil medicine $(n=153)$} \\
\hline Yes & 121 (79.I) \\
\hline No & $32(20.9)$ \\
\hline RT dose, mean $\pm s d(n=\mid 53)$ & $53.01 \pm 6.47$ \\
\hline \multicolumn{2}{|l|}{ Radiation modes $(n=153)$} \\
\hline Conventional fraction radiotherapy & $130(85.0)$ \\
\hline Accelerated hyperfractionation & $23(15.0)$ \\
\hline \multicolumn{2}{|l|}{ Second-line chemotherapy $(n=125)$} \\
\hline Yes & $85(68.0)$ \\
\hline No & $40(32.0)$ \\
\hline \multicolumn{2}{|l|}{ Survival time $(n=153)$} \\
\hline Median PFS (range) & II.0 (I.2-67.7) \\
\hline Median OS (range) & $23.3(6.0-70.2)$ \\
\hline
\end{tabular}


Table 2 Change of NLR and PLR at different stages of treatment

\begin{tabular}{clc}
\hline Value & Mean \pm sd & P-value \\
\hline NLR & $3.26 \pm 1.36$ & \\
$\mathrm{NLR}_{1}$ & $2.87 \pm 1.60$ & 0.016 \\
$\mathrm{NLR}_{2}$ & $8.98 \pm 9.96$ & $<0.01$ \\
$\mathrm{NLR}_{3}$ & $4.27 \pm 3.70$ & 0.499 \\
$\mathrm{NLR}_{1}$ & $2.87 \pm 1.60$ & \\
$\mathrm{NLR}_{2}$ & $8.98 \pm 9.96$ & $<0.01$ \\
$\mathrm{NLR}_{3}$ & $4.27 \pm 3.70$ & 0.012 \\
$\mathrm{NLR}_{2}$ & $8.98 \pm 9.96$ & \\
$\mathrm{NLR}_{3}$ & $4.27 \pm 3.70$ & $<0.01$ \\
$\mathrm{PLR}$ & $151.50 \pm 60.01$ & \\
$\mathrm{PLR}_{1}$ & $182.18 \pm 94.30$ & $<0.01$ \\
$\mathrm{PLR}_{2}$ & $308.49 \pm 252.23$ & $<0.01$ \\
$\mathrm{PLR}_{3}$ & $193.58 \pm 106.35$ & 0.026 \\
$\mathrm{PLR}_{1}$ & $182.18 \pm 94.30$ & \\
$\mathrm{PLR}_{2}$ & $308.49 \pm 252.23$ & $<0.01$ \\
$\mathrm{PLR}_{3}$ & $193.58 \pm 106.35$ & 0.508 \\
$\mathrm{PLR}_{2}$ & $308.49 \pm 252.23$ & $<0.01$ \\
$\mathrm{PLR}_{3}$ & $193.58 \pm 106.35$ &
\end{tabular}

Abbreviations: NLR, neutrophil-to-lymphocyte ratio at diagnosis; PLR, platelet-tolymphocyte ratio at diagnosis; sd, standard deviation; NLR, neutrophil-to-lymphocyte ratio after one cycle of chemotherapy; $\mathrm{NLR}_{2}$, neutrophil-to-lymphocyte ratio after thoracic radiotherapy; $\mathrm{NLR}_{3}$, neutrophil-to-lymphocyte ratio at progression; $\mathrm{PLR}_{\text {, }}$, platelet-to-lymphocyte ratio after one cycle of chemotherapy; $\mathrm{PLR}_{2}$, platelet-tolymphocyte ratio after thoracic radiotherapy; $\mathrm{PLR}_{3}$, platelet-to-lymphocyte ratio at progression.

for high-NLR group ( $P=0.027)$ and 93\% for low-PLR group versus $78.1 \%$ for high-PLR group $(P=0.041)$.

\section{Prognostic significance of NLR and PLR at diagnosis}

In the univariate analysis, high NLR, elevated alkaline phosphatase, high stage, and high NLR plus high PLR at diagnosis were significantly associated with both shorter OS and PFS. However, high PLR was associated only with shorter OS. Besides this, the administration of GM-CSF was not associated with better OS or PFS (Table 4). Therefore, all these factors were included in the subsequent multivariate analysis. The result of multivariate analysis showed that both high NLR at diagnosis and high stage were independent prognostic factors for shorter OS and PFS (Table 5).

The optimal cutoff values were 3.2 for NLR, with AUC of 0.723, and 122.7 for PLR, with AUC of 0.623. Based on these cutoff values, patients were stratified into two groups. Survival was analyzed using the Kaplan-Meier method with this stratification (Figure 1). The results showed that lowNLR group was significantly associated with better OS (low vs high, 31.0 months vs 18.0 months, $P<0.01)$ and better PFS (low vs high, 13.0 months vs 9.3 months, $P<0.01$ ). The cumulative 3 -year OS rates were $37.3 \%$ in low-NLR group and $14.3 \%$ in high-NLR group. The cumulative 3 -year PFS rates were $22.9 \%$ in low-NLR group and $8.6 \%$ in highNLR group. However, low-PLR group was significantly associated only with better OS (low vs high, 31.0 months vs 20.7 months, $P=0.036$ ). The cumulative 3 -year OS rates were $36.8 \%$ in low-PLR group and $20.8 \%$ in high-PLR group.

Since disease stage had a significantly differential distribution between NLR groups, we also analyzed the associations between NLR and survival based on disease stage at diagnosis (Figure 2). For LS SCLC, low-NLR group was significantly associated with better OS (low vs high, 33.7 months vs 24.5 months, $P=0.019$ ), but not for PFS (low vs high, 26.2 months vs 12.0 months, $P=0.052$ ). Similarly, for ES stage, low-NLR group was significantly associated with better OS (low vs high, 17.3 months vs 13.3 months, $P=0.03$ ), but not for PFS (low vs high, 8.7 months vs 8.1 months, $P=0.115$ ).

\section{Discussion}

In this study, NLR at diagnosis was an independent prognostic factor, and high NLR was significantly associated with shorter OS and PFS in SCLC patients who received standard therapy. As an index of circulating immune cells, NLR could reflect the balance between system inflammation and host immune response for tumor progression, which might account for its predictive value in clinical outcomes. Similar results were also observed in studies on different kinds of cancers, such as esophageal cancer, advanced gastric cancer, colorectal cancer, breast cancer, non-small-cell lung cancer, and epithelial ovarian cancer. Recently, Shao and Cai ${ }^{16}$ published a study that showed that high pretreatment NLR predicts recurrence and poor prognosis for combined SCLC. In their study, the cutoff value of NLR was 4.15 , which is comparable to 3.2 of our study. Though these two cutoff values were distinct, both these studies demonstrated a powerful prognostic value of pretreatment NLR in SCLC patients. In another study conducted by Xie et al, ${ }^{17} 938$ patients were enrolled including 555 ES SCLC and 383 LS SCLC patients. They found that elevated NLR was an independent prognostic factor for poor OS in ES SCLC patients, whereas elevated PLR was an independent prognostic factor for poor OS in LS SCLC patients. However, we demonstrated that elevated NLR was an independent prognostic factor for poor OS in both ES SCLC and LS SCLC patients. The small sample size of our study may account for the discrepancy between these two studies. The prognostic value of PLR is limited in our study. Though patients with low PLR at diagnosis showed obviously prolonged OS compared to high-PLR group in the 
Table 3 Clinical and laboratory features according to NLR and PLR at diagnosis

\begin{tabular}{|c|c|c|c|c|c|c|}
\hline Variables & $\begin{array}{l}\text { NLR }<3.2, \\
n=83\end{array}$ & $\begin{array}{l}\text { NLR } \geq 3.2, \\
n=70\end{array}$ & $P$-value & $\begin{array}{l}\text { PLR }<122.7 \\
n=57\end{array}$ & $\begin{array}{l}P L R \geq 122.7 \\
n=96\end{array}$ & $P$-value \\
\hline Age (range), years $(n=153)$ & $61(28-75)$ & $62(35-79)$ & 0.321 & 61 (28-78) & $62(35-79)$ & 0.621 \\
\hline $\operatorname{Sex}(n=153)$ & & & 0.121 & & & 0.465 \\
\hline Male & $60(72.3)$ & $58(82.9)$ & & $45(78.9)$ & $73(76.0)$ & \\
\hline Female & $23(27.7)$ & $12(17.1)$ & & $12(21.1)$ & $26(24.0)$ & \\
\hline Smoking status $(\mathrm{n}=\mid 53)$ & & & 0.218 & & & 0.853 \\
\hline Never smoker & $29(34.9)$ & I8 (25.7) & & $17(29.8)$ & $30(31.3)$ & \\
\hline Current or ex-smoker & $54(65.1)$ & $52(74.3)$ & & $40(70.2)$ & $66(68.7)$ & \\
\hline Stage $(n=153)$ & & & $<0.01$ & & & 0.009 \\
\hline LS & $58(69.9)$ & $27(38.6)$ & & $43(75.4)$ & $52(54.2)$ & \\
\hline ES & $25(30.1)$ & $43(61.4)$ & & $14(24.6)$ & $44(45.8)$ & \\
\hline KPS at diagnosis $(n=153)$ & & & 0.043 & & & 0.481 \\
\hline$\geq 80$ & $79(95.2)$ & $60(85.7)$ & & $53(93.0)$ & $86(89.6)$ & \\
\hline$<80$ & $4(4.8)$ & $10(14.3)$ & & $4(7.0)$ & $10(10.4)$ & \\
\hline $\mathrm{Hb}$ at diagnosis $(n=153)$ & & & 1.000 & & & 0.212 \\
\hline Normal $(\leq \mathrm{ULN})$ & $79(95.2)$ & $67(95.7)$ & & $55(97.4)$ & $87(90.6)$ & \\
\hline Low $(<$ LLN $)$ & $4(4.8)$ & $3(4.3)$ & & $2(2.6)$ & $9(9.4)$ & \\
\hline Alb at diagnosis $(n=\mid 36)$ & & & 0.005 & & & 0.132 \\
\hline Normal $(\leq \mathrm{ULN})$ & $65(83.3)$ & $36(62.1)$ & & $42(80.8)$ & $58(69.0)$ & \\
\hline Low $(<$ LLN $)$ & $13(16.7)$ & $22(37.9)$ & & $10(19.2)$ & $26(31.0)$ & \\
\hline $\mathrm{LDH}$ at diagnosis $(\mathrm{n}=136)$ & & & 0.373 & & & 0.647 \\
\hline Normal $(<$ ULN) & $45(57.7)$ & $29(50.0)$ & & $27(51.9)$ & $47(55.9)$ & \\
\hline High $(\geq \mathrm{ULN})$ & $33(42.3)$ & $29(50.0)$ & & $25(48.1)$ & $37(44.1)$ & \\
\hline ALP at diagnosis $(n=136)$ & & & 0.069 & & & 0.088 \\
\hline Normal $(<$ ULN) & $75(96.2)$ & $51(87.9)$ & & $51(98.1)$ & $75(89.3)$ & \\
\hline High $(\geq U L N)$ & $39(3.8)$ & $7(12.1)$ & & I (II.9) & $9(10.7)$ & \\
\hline PLR at diagnosis, mean $\pm s d(n=\mid 53)$ & $|27.08 \pm 4| .07$ & $180.47 \pm 66.04$ & $<0.01$ & & & \\
\hline NLR at diagnosis, mean $\pm s d(n=153)$ & & & & $2.64 \pm 0.99$ & $3.62 \pm 1.43$ & $<0.01$ \\
\hline Response for initial chemotherapy $(n=153)$ & & & 0.027 & & & 0.041 \\
\hline CR & $5(6.0)$ & $4(5.7)$ & & $2(3.5)$ & $2(2.1)$ & \\
\hline PR & $73(88.0)$ & $50(7 \mathrm{I} .4)$ & & $51(89.5)$ & $73(76.0)$ & \\
\hline SD & $3(3.6)$ & $10(14.3)$ & & $\mathrm{I}(\mathrm{I} .8)$ & $16(16.7)$ & \\
\hline PD & $2(2.4)$ & $6(8.6)$ & & $3(5.3)$ & $5(5.2)$ & \\
\hline Prophylactic cranial irradiation $(n=127)$ & & & 0.004 & & & $0.54 I$ \\
\hline Yes & $23(33.8)$ & $7(11.9)$ & & $13(26.5)$ & $17(2 \mid .8)$ & \\
\hline No & $45(66.2)$ & $52(88.1)$ & & $36(73.5)$ & $61(78.2)$ & \\
\hline Inflammation at diagnosis $(n=153)$ & & & 0.326 & & & 0.476 \\
\hline Yes & $18(21.7)$ & $20(28.6)$ & & $16(28.1)$ & $22(22.9)$ & \\
\hline No & $65(78.3)$ & $50(71.4)$ & & $41(71.9)$ & $74(77.1)$ & \\
\hline Stimulate neutrophil medicine $(n=153)$ & & & 0.346 & & & 0.705 \\
\hline Yes & $68(81.9)$ & $53(75.7)$ & & $46(80.7)$ & $75(78.1)$ & \\
\hline No & $15(18.1)$ & $17(24.3)$ & & II (19.3) & $21(21.9)$ & \\
\hline RT dose, mean $\pm s d(n=\mid 53)$ & $53.98 \pm 5.87$ & $51.87 \pm 6.99$ & 0.047 & $54.11 \pm 6.05$ & $52.36 \pm 6.65$ & 0.127 \\
\hline Radiation modes $(n=153)$ & & & 0.502 & & & 0.503 \\
\hline CFR & $72(86.7)$ & $58(82.9)$ & & $47(82.5)$ & $83(86.5)$ & \\
\hline AHF & II (I3.3) & $12(17.1)$ & & $10(17.5)$ & $13(13.5)$ & \\
\hline Second-line chemotherapy $(n=125)$ & & & 0.006 & & & 0.038 \\
\hline Yes & $48(80.0)$ & $37(56.9)$ & & $40(78.4)$ & $45(60.8)$ & \\
\hline No & $12(20.0)$ & $28(43.1)$ & & II (2I.6) & $29(39.2)$ & \\
\hline \multicolumn{7}{|l|}{ Survival time $(n=153)$} \\
\hline Median PFS (range) & I 3.0 (3.3-67.7) & $9.3(1.2-64.9)$ & 0.006 & II $.0(3.3-67.7)$ & $10.7(1.2-60.0)$ & 0.315 \\
\hline Median OS (range) & $31.0(6.8-70.2)$ & $18.0(6.0-64.9)$ & $<0.01$ & $31.0(8.9-70.2)$ & $20.7(6.0-68.7)$ & 0.015 \\
\hline
\end{tabular}

Note: Data shown as $\mathrm{n}(\%)$ unless indicated otherwise.

Abbreviations: NLR, neutrophil-to-lymphocyte ratio; PLR, platelet-to-lymphocyte ratio; LS, limited stage; ES, extensive stage; KPS, Karnofsky performance status; $\mathrm{Hb}$, hemoglobin; ULN, upper limit of normal; LLN, lower limit of normal; Alb, albumin; LDH, lactate dehydrogenase; ALP, alkaline phosphatase; sd, standard deviation; $\mathrm{CR}$, complete response; PR, partial response; SD, stable disease; PD, progressive disease; RT, radiation therapy; CFR, conventional fraction radiotherapy; AHF, accelerated hyperfractionation; PFS, progression-free survival; OS, overall survival. 
Table 4 Univariate analysis for OS and PFS

\begin{tabular}{|c|c|c|c|c|}
\hline \multirow[t]{2}{*}{ Variables } & \multicolumn{2}{|l|}{ OS } & \multicolumn{2}{|l|}{ PFS } \\
\hline & $\chi^{2}$ & $P$-value & $x^{2}$ & $P$-value \\
\hline \multicolumn{5}{|c|}{ Smoking status } \\
\hline \multicolumn{5}{|c|}{ No } \\
\hline Yes & 4.707 & 0.03 & 5.43 & 0.02 \\
\hline \multicolumn{5}{|l|}{ Stage } \\
\hline \multicolumn{5}{|l|}{ LS } \\
\hline ES & 24.34 & $<0.01$ & 26.11 & $<0.01$ \\
\hline \multicolumn{5}{|c|}{ Stimulate neutrophil medicine } \\
\hline \multicolumn{5}{|l|}{ Yes } \\
\hline No & 0.141 & 0.707 & 0.541 & 0.462 \\
\hline \multicolumn{5}{|c|}{ NLR at diagnosis } \\
\hline \multicolumn{5}{|c|}{ Low } \\
\hline High & $18.5 \mid$ & $<0.01$ & 13.83 & $<0.01$ \\
\hline \multicolumn{5}{|c|}{ PLR at diagnosis } \\
\hline \multicolumn{5}{|c|}{ Low } \\
\hline High & 4.38 & 0.036 & 2.05 & 0.152 \\
\hline \multicolumn{5}{|c|}{ NLR combined with PLR at diagnosis } \\
\hline \multicolumn{5}{|c|}{ Both low } \\
\hline One high & 0.236 & 0.627 & 0.16 & 0.900 \\
\hline Both high & 16.635 & $<0.01$ & 9.99 & 0.002 \\
\hline \multicolumn{5}{|c|}{ ALP at diagnosis } \\
\hline \multicolumn{5}{|c|}{ Low } \\
\hline High & 8.32 & 0.004 & 4.13 & 0.042 \\
\hline \multicolumn{5}{|c|}{ NLR after one cycle of chemotherapy } \\
\hline Low & & 0.536 & 0.08 & 0.779 \\
\hline High & 0.38 & & & \\
\hline \multicolumn{5}{|c|}{ NLR after TRT } \\
\hline \multicolumn{5}{|c|}{ Low } \\
\hline High & I.II & 0.293 & 0.88 & 0.348 \\
\hline \multicolumn{5}{|c|}{ NLR at disease progression } \\
\hline \multicolumn{5}{|c|}{ Low } \\
\hline High & 0.45 & 0.504 & 0.04 & 0.846 \\
\hline \multicolumn{5}{|c|}{ PLR after one cycle of chemotherapy } \\
\hline \multicolumn{5}{|c|}{ Low } \\
\hline High & 0.86 & 0.354 & 0.75 & 0.386 \\
\hline \multicolumn{5}{|c|}{ PLR after TRT } \\
\hline \multicolumn{5}{|l|}{ Low } \\
\hline High & 0.01 & 0.924 & 0.47 & 0.49 \\
\hline PLR at disea & gression & & & \\
\hline Low & & & & \\
\hline High & 0.32 & 0.570 & 1.46 & 0.226 \\
\hline
\end{tabular}

Abbreviations: OS, overall survival; PFS, progression-free survival; LS, limited stage; ES, extensive stage; NLR, neutrophil-to-lymphocyte ratio; PLR, platelet-tolymphocyte ratio; ALP, alkaline phosphatase; TRT, thoracic radiotherapy. univariate analysis, PLR did not show prognostic significance in the multivariate analysis. Nevertheless, the prognostic value of PLR has been demonstrated in some other studies related to hepatocellular carcinoma, soft-tissue sarcoma, and non-small-cell lung cancer. ${ }^{18-20} \mathrm{Gu}$ et al demonstrated that high PLR predicted poor OS and poor PFS in a meta-analysis that included eleven studies with 3,430 patients. However, subgroup analysis showed that the prognostic role of PLR was detected only in Caucasians but not in Asians. The ethnic heterogenicity may be the potential reason. This discrepancy might be caused by the small sample size of our study, in which the low-PLR group only included 57 patients.

Many studies have demonstrated that tumor-associated inflammation plays a critical role in tumor progression, and the functions of tumor-associated neutrophils are very important. Although these neutrophils have two opposite roles, antitumorigenic and protumorigenic, ${ }^{21}$ several studies have suggested that they mostly have the protumorigenic function that contributes to tumor growth and immunosuppression, and the depletion of these neutrophils could inhibit tumor growth. ${ }^{22-24}$ Furthermore, Spicer et $\mathrm{al}^{25}$ found that neutrophils could facilitate cancer cell adhesion within liver sinusoids and promote metastasis via Mac-1-mediated interactions with cancer cells. The protumorigenic role of neutrophils has also been demonstrated in melanoma. Slattery and Cheng $^{26}$ found that neutrophils could influence melanoma cell adhesion and migration through the endothelium via Mac-1/ICAM-1 interactions. In addition, it is known that during antitumor immune response, lymphocytes could inhibit tumor proliferation and migration. Therefore, the NLR value, based on absolute neutrophil count to absolute lymphocyte count ratio, has prognostic significance in many types of tumors. ${ }^{8-13}$ The mechanisms for the formation of specialized microenvironments ("niches") during metastasis, which consist of host cells and disseminated tumor cells, are still not clear. Labelle et $\mathrm{al}^{27}$ found that platelet-derived signals for chemokine secretion played an important role in

Table 5 Multivariate analysis for OS and PFS

\begin{tabular}{|c|c|c|c|c|c|c|}
\hline \multirow[t]{2}{*}{ Parameter } & \multicolumn{3}{|l|}{ OS } & \multicolumn{3}{|l|}{ PFS } \\
\hline & HR & $95 \% \mathrm{Cl}$ & $P$-value & HR & $95 \% \mathrm{Cl}$ & $P$-value \\
\hline \multicolumn{7}{|c|}{ NLR at diagnosis } \\
\hline Low & Reference & & & Reference & & \\
\hline High & $\mathrm{I} .724$ & I.116-2.663 & 0.014 & 1.589 & $1.049-2.406$ & 0.029 \\
\hline \multicolumn{7}{|l|}{ Stage } \\
\hline LS & Reference & & & Reference & & \\
\hline ES & 1.926 & $1.248-2.973$ & 0.003 & 2.168 & $1.398-3.360$ & 0.001 \\
\hline
\end{tabular}

Abbreviations: OS, overall survival; PFS, progression-free survival; HR, hazard ratio; Cl, confidence interval; NLR, neutrophil-to-lymphocyte ratio; LS, limited stage; ES, extensive stage. 

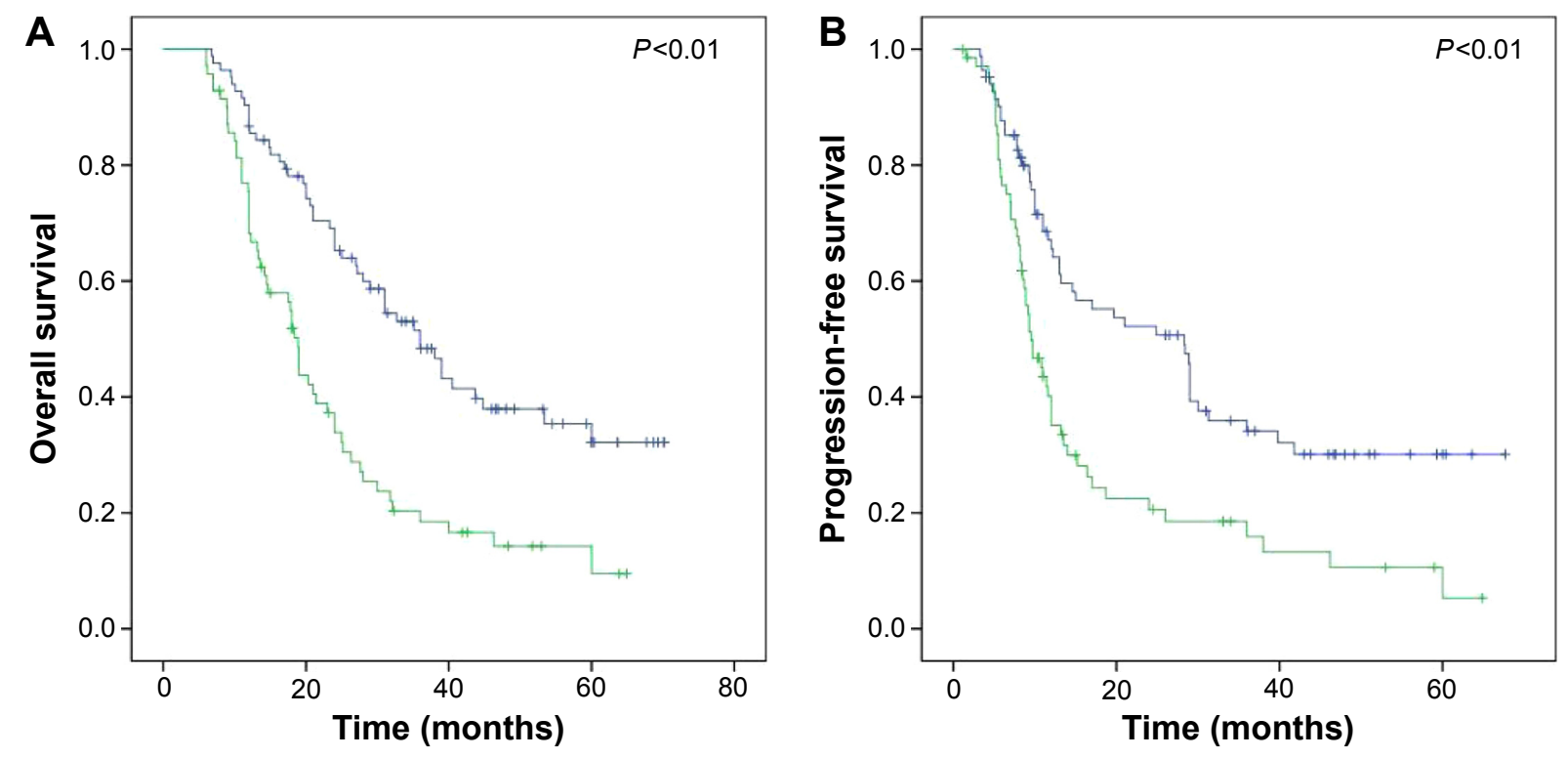

NLR
$\neg<3.2(\mathrm{n}=83) \quad-\geq 3.2(\mathrm{n}=70) \quad+<3.2$ censored $+\geq 3.2$ censored
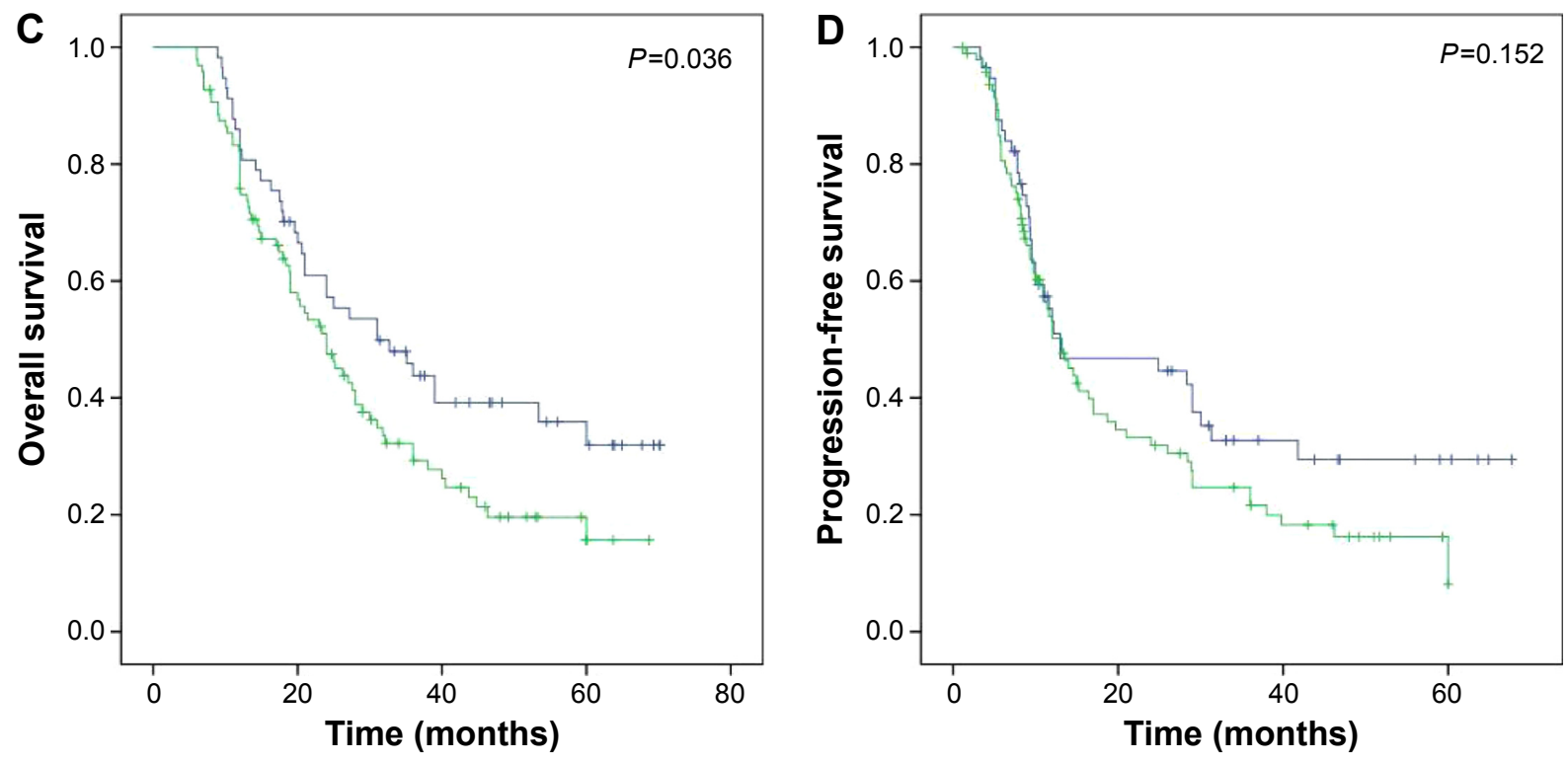

\begin{tabular}{l} 
PLR \\
$\neg<122.7(\mathrm{n}=57) \quad-\geq 122.7(\mathrm{n}=96)+<122.7$ censored $+\geq 122.7$ censored \\
\hline
\end{tabular}

Figure I Survival for SCLC patients according to NLR and PLR stratification.

Notes: (A) OS according to NLR. Solid blue - NLR $<3$.2, solid green - NLR $\geq 3.2$. (B) PFS according to NLR. Solid blue - NLR $<3.2$, solid green - NLR $\geq 3$.2. (C) OS according to PLR. Solid blue - PLR $<122.7$, solid green - PLR $\geq 1$ 22.7. (D) PFS according to PLR. Solid blue - PLR $<122.7$, solid green - PLR $\geq 122.7$.

Abbreviations: SCLC, small-cell lung cancer; NLR, neutrophil-to-lymphocyte ratio; PLR, platelet-to-lymphocyte ratio; OS, overall survival; PFS, progression-free survival.

recruiting granulocytes to tumor cells, which then formed "early metastatic niches" that accelerated tumor progression. Although another two studies confirmed the prognostic value of PLR in hepatocellular carcinoma and soft-tissue sarcoma, ${ }^{18,19}$ we did not find the independent prognostic value of PLR for predicting clinical outcomes of SCLC patients in our study.
As shown in Table 2, NLR changed significantly along with disease and treatment progression. It decreased after one cycle of chemotherapy compared to the time of diagnosis and then significantly increased after TRT. At disease progression point, NLR decreased again but was still higher than the level of diagnosis. Since SCLC is highly responsive to initial CRT, the reduction of NLR after one cycle 

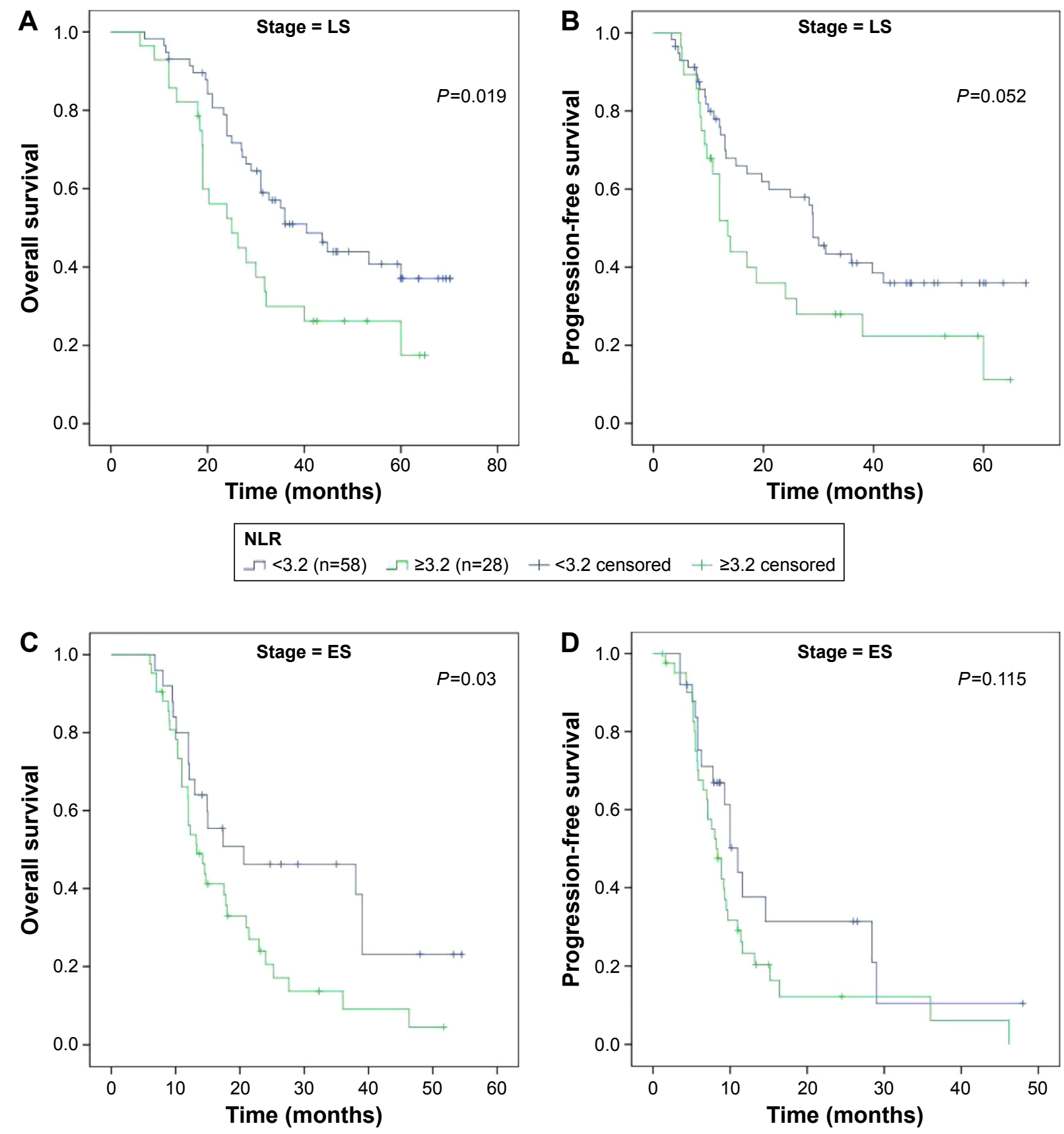

\begin{tabular}{|l|l|}
\hline NLR \\
$\neg<3.2(n=25) \quad-\geq 3.2(n=42) \quad+<3.2$ censored $\quad+\geq 3.2$ censored \\
\hline
\end{tabular}

Figure 2 Survival in LS and ES patients according to NLR stratification.

Notes: (A) OS in LS patients according to NLR. Solid blue - NLR $<3.2$, solid green - NLR $\geq 3.2$. (B) PFS in LS patients according to NLR. Solid blue - NLR $<3.2$, solid green $-N L R \geq 3.2$. (C) OS in ES patients according to NLR. Solid blue $-N L R<3$.2, solid green $-N L R \geq 3.2$. (D) PFS in ES patients according to NLR. Solid blue - NLR $<3.2$, solid green $-\mathrm{NLR} \geq 3.2$.

Abbreviations: LS, limited stage; ES, extensive stage; NLR, neutrophil-to-lymphocyte ratio; OS, overall survival; PFS, progression-free survival; ES, extensive stage.

of chemotherapy was reasonable. Although we examined response for initial chemotherapy after two cycles of chemotherapy, it should still reflect the tumor burden after one cycle of chemotherapy to some degree. We found that lowNLR group had higher response rate (94.0\%) than high-NLR group (77.1\%). At disease progression point, NLR increased again, which suggested heavier tumor burden and relatively more immunosuppressive status compared to the time of diagnosis. However, the peak value of NLR occurred after TRT, which might be opposite to our understanding, as TRT is an optimal treatment method for SCLC patients. After analyzing original data, we found that lymphocytopenia after 
TRT was the main cause for the peak of NLR. Therefore, the prognostic value of NLR after TRT should be further studied. Composite stratifications together with other biomarkers may be a good way to go. In summary, we found that NLR was a good index reflecting tumor burden and host immune status, and it could help evaluating treatment response and monitoring disease recurrence.

Compared with high-NLR group, low-NLR group had significantly prolonged OS and PFS. However, high-NLR group had a lower ratio (40.0\%) of LS SCLC patients than low-NLR group (69.9\%), which is a selection bias that should not be ignored. Therefore, we analyzed the prognostic value of NLR using another stratification based on disease stage. For OS, low-NLR group was associated with prolonged OS either for LS SCLC or for ES SCLC, compared with highNLR group. But for PFS, the difference was not significant for both LS SCLC and ES SCLC. The reason could be that the association between disease stage and NLR stratification for PFS is closer than that for OS in multivariate analysis. In spite of this, NLR is still suggested as an independent prognostic index for survival in our study.

One of the limitations of our study is its retrospective characterization, where we could not control the potential confounding factors. A further limitation is the small sample size, and hence a large sample size study is needed to verify our results. Although limitations exist, we cannot ignore the value of this study, which suggests NLR as an effective index to predict clinical outcomes of SCLC patients.

\section{Conclusion}

As an inexpensive and readily available index, NLR has its advantage in reflecting associations between tumor-associated inflammation and tumor burden. Our study highlighted the prognostic value of NLR at diagnosis for the survival of SCLC patients.

\section{Disclosure}

The authors report no conflicts of interest in this work.

\section{References}

1. Govindan R, Page N, Morgensztern D, et al. Changing epidemiology of small-cell lung cancer in the United States over the last 30 years: analysis of the surveillance, epidemiologic, and end results database. J Clin Oncol. 2006;24(28):4539-4544.

2. van Meerbeeck JP, Fennell DA, De Ruysscher DK. Small-cell lung cancer. Lancet. 2011;378(9804):1741-1755.

3. Früh M, De RD, Popat S, et al. Small-cell lung cancer (SCLC): ESMO clinical practice guidelines for diagnosis, treatment and follow-up. Ann Oncol. 2013;24(suppl 6):vi99-vi105.

4. Morabito A, Carillio G, Daniele G, et al. Treatment of small cell lung cancer. Crit Rev Oncol Hematol. 2014;91(3):257-270.

5. Grivennikov SI, Greten FR, Karin M. Immunity, inflammation, and cancer. Cell. 2010;140(6):883-899.
6. de Visser KE, Eichten A, Coussens LM. Paradoxical roles of the immune system during cancer development. Nat Rev Cancer. 2006; 6(1):24-37.

7. Dannenberg AJ, Kotha S. Targeting cyclooxygenase-2 in human neoplasia: rationale and promise. Cancer Cell. 2003;4(6):431-436.

8. Walsh SR, Cook EJ, Goulder F, Justin TA, Keeling NJ. Neutrophillymphocyte ratio as a prognostic factor in colorectal cancer. $J$ Surg Oncol. 2005;91(3):181-184.

9. Yamanaka T, Matsumoto S, Teramukai S, Ishiwata R, Nagai Y, Fukushima M. The baseline ratio of neutrophils to lymphocytes is associated with patient prognosis in advanced gastric cancer. Oncology. 2007; 73(3-4):215-220.

10. Sarraf KM, Belcher E, Raevsky E, Nicholson AG, Goldstraw P, Lim E. Neutrophil/lymphocyte ratio and its association with survival after complete resection in non-small cell lung cancer. $J$ Thorac Cardiovasc Surg. 2009;137(2):425-428.

11. Azab B, Bhatt VR, Phookan J, et al. Usefulness of the neutrophil-tolymphocyte ratio in predicting short- and long-term mortality in breast cancer patients. Ann Surg Oncol. 2012;19(1):217-224.

12. Cho HB, Hur HW, Sang WK, et al. Pre-treatment neutrophil to lymphocyte ratio is elevated in epithelial ovarian cancer and predicts survival after treatment. Cancer Immunol Immunother. 2009;58(1):15-23.

13. Sharaiha RZ, Halazun KJ, Mirza F, et al. Elevated preoperative neutrophil:lymphocyte ratio as a predictor of postoperative disease recurrence in esophageal cancer. Ann Surg Oncol. 2011;18(12):3362-3369.

14. Cummings M, Merone L, Keeble C, et al. Preoperative neutrophil: lymphocyte and platelet:lymphocyte ratios predict endometrial cancer survival. Br J Cancer. 2015;113(2):311-320.

15. Eisenhauer EA, Therasse P, Bogaerts J, et al. New response evaluation criteria in solid tumours: revised RECIST guideline (version 1.1). Eur J Cancer. 2009;45(2):228-247.

16. Shao N, Cai Q. High pretreatment neutrophil-lymphocyte ratio predicts recurrence and poor prognosis for combined small cell lung cancer. Clin Trans Oncol. 2015;17:1-7.

17. Xie D, Marks R, Zhang M, et al. Nomograms predict overall survival for patients with small-cell lung cancer incorporating pretreatment peripheral blood markers. J Thorac Oncol. 2015;10(8):1213-1220.

18. Li X, Chen ZH, Ma XK, et al. Platelet-to-lymphocyte ratio acts as a prognostic factor for patients with advanced hepatocellular carcinoma. Tumor Biol. 2015;36(4):2263-2269.

19. Que Y, Qiu H, Li Y, et al. Preoperative platelet-lymphocyte ratio is superior to neutrophil-lymphocyte ratio as a prognostic factor for softtissue sarcoma. BMC Cancer. 2015;15(1):648.

20. Gu X, Sun S, Gao XS, et al. Prognostic value of platelet to lymphocyte ratio in non-small cell lung cancer: evidence from 3,430 patients. Sci Rep. 2016;6:23893.

21. Fridlender ZG, Sun J, Kim S, et al. Polarization of tumor-associated neutrophil phenotype by TGF-beta: "N1" versus "N2" TAN. Cancer Cell. 2009;16(3):183-194.

22. Pekarek LA, Starr BA, Toledano AY, Schreiber H. Inhibition of tumor growth by elimination of granulocytes. J Exp Med. 1995;181(1): 435-440.

23. Tazawa H, Okada F, Kobayashi T, et al. Infiltration of neutrophils is required for acquisition of metastatic phenotype of benign murine fibrosarcoma cells: implication of inflammation-associated carcinogenesis and tumor progression. Am J Pathol. 2003;163(6):2221-2232.

24. Schmielau J, Finn OJ. Activated granulocytes and granulocyte-derived hydrogen peroxide are the underlying mechanism of suppression of t-cell function in advanced cancer patients. Cancer Res. 2001;61(12): 4756-4760.

25. Spicer JD, Braedon MD, Cools-Lartigue JJ, et al. Neutrophils promote liver metastasis via Mac-1-mediated interactions with circulating tumor cells. Cancer Res. 2012;72(16):3919-3927.

26. Slattery MJ, Cheng D. Neutrophils influence melanoma adhesion and migration under flow conditions. Int J Cancer. 2003;106(5):713-722.

27. Myriam L, Shahinoor B, Hynes RO. Platelets guide the formation of early metastatic niches. Proc Natl Acad Sci U S A. 2014;111(30): E3053-E3061. 


\section{Publish your work in this journal}

OncoTargets and Therapy is an international, peer-reviewed, open access journal focusing on the pathological basis of all cancers, potential targets for therapy and treatment protocols employed to improve the management of cancer patients. The journal also focuses on the impact of management programs and new therapeutic agents and protocols on

patient perspectives such as quality of life, adherence and satisfaction. The manuscript management system is completely online and includes a very quick and fair peer-review system, which is all easy to use. Visit http://www.dovepress.com/testimonials.php to read real quotes from published authors.

Submit your manuscript here: http://www.dovepress.com/oncotargets-and-therapy-journal 